\title{
Regard sur les dimensions sous-jacentes dans les épreuves de conscience morphologique
}

\author{
Rosalie Bourdages ${ }^{1,2^{*}}$, et Denis Foucambert ${ }^{1}$ \\ ${ }^{1}$ Université du Québec à Montréal, C.P. 8888, succursale Centre-Ville, Montréal, Québec, H3C 3P8, \\ Canada \\ ${ }^{2}$ Haute école pédagogique du canton de Vaud, Avenue de Cour 33, CH-1014, Lausanne, Suisse
}

\begin{abstract}
Résumé. Malgré l'importance de la conscience morphologique pour le développement d'habiletés de haut niveau indispensables au succès scolaire (Apel, Diehm et Apel, 2013; Kieffer et al. 2016; Tighe et Schatschneider; 2016), il y a une réelle confusion dans la littérature sur le plan de la mesure de ce construit, en ce sens que les tâches utilisées pour le circonscrire font intervenir des habiletés d'ordre cognitif et linguistique extrêmement variées (Deacon, Parrila et Kirby, 2008). En nous basant sur les épreuves traditionnellement utilisées pour mesurer la conscience morphologique, nous avons construit six tâches qui mobilisent à la fois des unités (morphèmes flexionnel ou dérivationnel), des opérations (suppression ou ajout) et des types de contrôle (implicites ou explicites) divers (Apel, 2014). Nos données ont été récoltées auprès d'enfants québécois et français de 8 à 10 ans. Les résultats, issus d'analyses factorielles confirmatoires (AFC), mettent en relief l'importance du type d'opérations cognitives effectuées comme facteur permettant de discriminer les épreuves entre elles.
\end{abstract}

\begin{abstract}
Looking at the underlying dimensions in morphological awareness tasks. Despite the importance of morphological awareness for the development of high-level skills essential to academic success (Apel, Diehm et Apel, 2013; Kieffer et al. 2016; Tighe et Schatschneider; 2016), there is confusion in the literature regarding the measurement of this construct, in that the tasks used to assess it involve extremely varied cognitive and linguistic skills (Deacon, Parrila and Kirby, 2008). Based on the tasks traditionally used to measure morphological awareness, we constructed six tasks who mobilize various units (flexional/derivational), operations (deletion/addition) and levels of control (implicit/explicit) (Apel, 2014). Our data was collected from 8 to 10 years old children from Quebec and France. To understand how the tasks are grouped, we performed confirmatory factor analyzes (CFA), which highlight the importance of the type of cognitive operations performed as a factor that makes it possible to explain variation between the tasks.
\end{abstract}

* Corresponding author : bourdages.rosalie@uqam.ca 


\section{Introduction}

La conscience morphologique se définit comme l'identification et la manipulation conscientes de la structure morphologique des mots (Carlisle, 1995; Gombert, 1990). Les unités morphologiques des langues sont porteuses de sens, et permettent d'établir les relations sémantiques ou syntaxiques entre les mots et les propositions (Chomsky et Halle, 1968; Carlisle, 1995). Les morphèmes flexionnels et dérivationnels constituent les deux types de morphèmes liés du français, c'est-à-dire qu'ils n'existent pas comme base ou comme mot indépendants. Alors que les morphèmes flexionnels fournissent des informations grammaticales additionnelles sans modifier la catégorie de la base, les morphèmes dérivationnels peuvent modifier la catégorie grammaticale et/ou la signification sémantique de la base (Bloomfield, 1933). L'analyse du mot inévitable, par exemple, permet de bien exprimer le caractère compositionnel de la morphologie: ce mot multimorphémique est formé à partir de la base verbale évit(er), à laquelle est ajouté le suffixe dérivationnel -able ('caractère de ce qui est X'), formant la nouvelle base évitable, à laquelle se greffe le préfixe in- ('n'est pas X'). On comprend très bien, à travers cet exemple, que le tout est ici bel et bien la somme de ses parties, et combien l'accès au sens se fait par l'entremise d'une analyse plus ou moins réussie et plus ou moins automatique de chacune des composantes du mot. Il n'est ainsi pas étonnant que l'habileté à repérer et à opérationnaliser les plus petites unités de sens de la langue soit réputée contribuer aux performances en lecture (Kieffer et al., 2016; Manolitsis, Grigorakis et Georgiou, 2017; Casalis et Louis-Alexandre, 2000) et en en écriture (Green et al., 2003).

La littérature sur la conscience morphologique est pourtant marquée par d'importantes disparités sur le plan de sa mesure (Berthiaume, Besse et Daigle, 2010). Les revues méthodologiques qui ont été effectuées sur ce construit théorique signalent en effet unanimement la diversité des tâches qui sont utilisées pour le circonscrire (Apel, Diehm et Apel, 2013; Apel, 2014; Deacon, Parrila et Kirby, 2008; Casalis, Mathiot, Bécavin et Colé, 2003; Berthiaume, Besse et Daigle, 2010). Les tâches peuvent être présentées oralement ou par écrit, et les réponses peuvent être recueillies dans l'une ou l'autre de ces modalités. Le morphème peut être flexionnel ou dérivationnel (préfixe ou suffixe), doit également être contrôlé pour sa transparence sur les plans phonologique et orthographique et pour sa fréquence. Les opérations cognitives et les niveaux de contrôle qui sont déployés dans le cadre de ces épreuves sont également différents (Berthiaume, Besse et Daigle, 2010). Il est donc raisonnable de penser que la pluralité des mesures qui caractérise les études effectuées sur la conscience morphologique pourrait expliquer, au moins en partie, les écarts observés dans la prédiction d'habiletés de haut niveau essentielles au succès scolaire, notamment les compétences en lecture et en écriture (Apel, Diehm et Apel, 2013; Tighe et Schatschneider; 2016). Afin d'aller au-delà des confusions causées par ce problème méthodologique, nous présenterons, dans le cadre de cette étude, les dimensions sous-jacentes pouvant représenter des facteurs de variation dans la performance aux épreuves de conscience morphologique. Nous proposerons une analyse de données à l'aide de modélisations statistiques permettant de faire la lumière sur les habiletés qui sont mises en œuvre dans les différentes épreuves de conscience morphologique.

\section{Taxonomie des épreuves de conscience morphologique}

Il est important de considérer les facteurs de variation dans les épreuves qui sont réputées mesurer le même construit théorique - c'est-à-dire la conscience morphologique, dans le cas qui nous concerne. La conscience morphologique est évaluée à l'aide de différentes épreuves, dont les habiletés mobilisées pour les réussir sont variées (Casalis, Mathiot, 
Bécavin et Colé, 2003; Muse, 2005; Berthiaume, Besse et Daigle, 2010). On retrouve notamment dans la littérature des épreuves de jugement de relation de mots, de jugement de grammaticalité, d'identification de l'erreur suivie d'une correction, de réplication de l'erreur (pour voir si le sujet est capable de reproduire l'agrammaticalité sur une phrase correcte), de production de dérivés pour une même base, d'identification d'affixes dans pseudo-mots (ex. dans mouteur) ou encore de complétion de phrases à l'aide de pseudomots (Kieffer, Petscher, Proctor et Silverman, 2016).

\subsection{Modalités de présentation}

Comme le mentionnent Deacon, Parrila \& Kirby (2008), qui ont étudié la conscience morphologique chez les dyslexiques, les tâches se différencient sur le plan de la modalité de leur présentation à l'élève (input), qui peut fort bien être différente de la modalité de la réponse demandée (output). Les habiletés en décodage, notamment, sont susceptibles d'influencer les résultats dans les temps de réponse, et même dans la qualité de la réponse fournie. L'orthographe a par contre l'avantage de maintenir les indices morphologiques au sein des items, ce qui pourrait représenter un avantage pour certain-e-s participant-e-s. Il est important, comme le soulignent Deacon, Parrila \& Kirby (2008), de rappeler que dans le cas où les tâches sont présentées et recueillies à l'écrit, les morphèmes constituent aussi des unités orthographiques : il s'agit donc d'un facteur qui doit être contrôlé ou analysé.

\subsection{Types de morphèmes (unités)}

Le traitement des dérivations est réputé être plus complexe que celui des flexions, car le procédé de dérivation implique souvent une altération phonologique et orthographique de la base, en plus de rendre possible une transformation de la catégorie du mot (Tighe et Schatschneider, 2015; Casalis et al., 2003; Tyler et Nagy, 1989). L'acquisition des morphèmes flexionnels et dérivationnels est aussi marquée par des différences sur le plan développemental. Alors que les flexions sont maîtrisées plus tôt, souvent dans les premières années de l'école primaire (Berko, 1958; Roy et Labelle, 2007), la morphologie dérivationnelle continue de se développer au-delà de cette période, chez certains élèves (Roy et Labelle, 2007). Plusieurs tâches de conscience morphologique n'impliquent que des morphèmes flexionnels ou des morphèmes dérivationnels; d'autres impliquent une combinaison des deux. Cependant, il reste à évaluer de façon empirique si ces deux types d'unités représentent des facettes distinctes de la conscience morphologique.

\subsection{Opérations}

Apel (2014) dénombre cinq opérations ou manipulations déployées dans les épreuves dont il brosse le portrait. Les tâches de jugement exigent des participant-e-s une prise de décision sur la relation sémantique entre deux mots. Certaines tâches demandent de segmenter des mots en plusieurs morphèmes, ou de mélanger (blending) des morphèmes pour former des mots multimorphémiques, reprenant des opérations que nous retrouvons dans les tâches de dérivation et de décomposition. Les tâches d'analogie demandent de compléter une série de mots en imitant la structure dans une première série de mots (ex. fleur : fleuriste, garage :

). L'identification d'affixes dans des pseudo-mots correspond pour Apel (2014) à un autre type d'opération. Dans le cadre des tâches d'identification d'affixes, une liste de pseudo-mots est fournie, et on demande aux participant-e-s d'enclercler les suffixes qu'ils parviennent à repérer (Apel, Diehm et Apel, 2013).

La typologie des différentes opérations des tâches de conscience morphologique relevées 
dans le travail d'Apel (2014) ne sont pas reprises intégralement dans la présente recherche : nous avons plutôt cherché à les reconceptualiser de façon binaire - ajout et suppression -, en fonction de notre compréhension de ce qui est demandé aux participants de faire pour réussir la tâche. Les détails sont fournis dans la section 3.2.

\subsection{Niveaux de contrôle}

Il faut impérativement distinguer les épreuves qui demandent une compréhension ou une réception de morphèmes de celles qui en nécessitent la production (Casalis et al., 2003; Deacon, Parrila \& Kirby, 2008). Dans les épreuves de réception, par exemple lorsqu'on demande à l'élève de choisir, parmi quelques formes dérivées (flexionnelle ou dérivationnelle), celle qui convient le mieux, le niveau de contrôle est dit implicite (Deacon, Parrila \& Kirby, 2008; Berthiaume, Besse et Daigle, 2010). Ce type de tâche se différencie d'une situation de production, dans laquelle l'enfant est amené à donner une forme dérivée lorsqu'on lui fournit une base (Berko, 1958; Carlisle, 1995), ce qui correspond, dans notre terminologie, à un niveau de contrôle explicite.

\section{Synthèse et objectifs de recherche}

Puisque les tâches représentant la conscience morphologique sont si variées, il est légitime de se demander ce qu'elles mesurent réellement (Berthiaume, Besse et Daigle, 2010). Comme le problématise justement Apel (2014, p. 204), la question est de connaitre à quel point les différentes épreuves qui définissent la conscience morphologique sont interchangeables et équivalentes, et comment chacune d'elles mesure des aspects différenciés de ce construit intrinsèquement multifactoriel. La conscience morphologique est encore peu étudiée dans une perspective de variables latentes ou de validité de construit (construct validity) (Deacon, Parrila et Kirby, 2008). Bien que certaines études se soient penchées sur les dimensions sous-jacentes à la conscience morphologique en anglais (Muse, 2005; Spencer et al., 2015; Tighe \& Schatschneider, 2015) et en arabe (Tibi et Kirby 2017), notre étude est à notre connaissance la première à le faire avec un échantillon de francophones. Ainsi, il est légitime de se demander ce que mesurent les différentes tâches de conscience morphologique en français. Y a-t-il moyen de mieux les comprendre, de mieux les définir? Ces tâches opérationnalisent-elles des habiletés d'ordre cognitif? Dans quelle mesure sont-elles tributaires de l'unité linguistique qu'elles mettent en œuvre ? Finalement, la conscience morphologique constitue-t-elle un construit unidimensionnel, ou met-elle en jeu plusieurs dimensions sous-jacentes?

\section{Méthode}

\subsection{Participants}

Afin de répondre à ces questions, nous avons mis en place une recherche expérimentale effectuée dans 4 classes différentes. Quatre-vingt-six sujets normolecteurs de 8 à 10 ans (moyenne d'âge $=9$ ans, 5 mois; écart type $=6,4$ mois) fréquentant l'école primaire ont participé à notre étude. On dénombre 59 Québécois et 27 Français. Nous avons pris soin de bien délimiter notre échantillon, en ne sélectionnant que des élèves ayant le français comme langue maternelle, puis en excluant les élèves ayant un diagnostic de TDAH ou de dyslexie. 
Les participant-e-s ont réalisé six tâches de conscience morphologique. Les passations se sont déroulées en trois séances subséquentes de cinquante minutes chacune, séparées par un maximum d'une semaine. Conséquemment, toutes les passations ont eu lieu dans le local informatique des deux écoles, et tous les élèves étaient assignés à un poste. Chaque passation était précédée d'une explication de quelques minutes, puis d'une période d'essai effectuée directement à l'aide d'un logiciel spécialement confectionné pour l'occasion. En effet, afin de bien délimiter l'objet à l'étude et l'effet indésirable qu'un transfert d'une modalité à une autre aurait pu occasionner, toutes les épreuves se sont déroulées à l'écrit, sur support informatique, plus spécifiquement à l'aide du logiciel Logix $@$ (Foucambert et Bourdages, 2017) - qui a facilité les passations et optimalisé la collecte et le codage des données.

\subsection{Constitution du matériel expérimental}

Au vu des différences sur le plan de la constitution des items dans les études précédentes, la présente étude a tenté de ramener les facteurs confondants à un minimum. Ainsi, dans la mesure où les connaissances du vocabulaire ne sont pas l'objet qui doit être mesuré dans notre expérimentation sur la conscience morphologique, la fréquence des items a été contrôlée en n'admettant que des lemmes très fréquents et fréquents, selon le logiciel Antidote $\subset$ (version 9.2). Nous avons aussi contrôlé les items (bases et affixes) sur le plan de la transparence : les transformations que l'élève doit effectuer pour arriver aux bonnes réponses n'entrainent pas de changement phonologique, et souvent aucun changement orthographique, bien que la construction très stricte de nos items ait parfois occasionné des changements orthographiques minimaux (maximum deux graphèmes), notamment dans l'épreuve de dérivation. Les épreuves ont été construites avec le souci de contrebalancer les différents formats d'items - en délimitant la nature des bases et des affixes : par exemple, une base nominale où l'objectif est d'apposer un suffixe formant des adjectifs correspond à un format $(\mathrm{N}>\mathrm{Adj})$. Toutes les réponses recueillies ont été codées selon leur qualité (de 0 à 4), puis ramenées à un score binaire (0-1). Nous présentons ci-bas les six épreuves utilisées dans le cadre de cette recherche.

\subsubsection{Paires de mots}

Dans cette épreuve, on demande à l'élève de prendre une décision sur la relation sémantique entre les deux mots qui lui sont présentés (Spencer et al., 2015; Carlisle \& Nomanbhoy, 1993; Colé, Royer, Leuwers \& Casalis, 2004; Roy et Labelle, 2007; Duncan, Casalis \& Colé, 2009; Allard, 2008). Le matériel est constitué de douze paires de mots ayant une base morphologique commune et de douze autres paires de mots reliés uniquement formellement (phonologiquement et orthographiquement), et qui sont pseudosuffixés, pour un total de 24 items. Parmi les douze vraies paires, six sont sous le format «Verbe, Nom (Base + suffixe)» (ex. glisser, glissade) et six autres sont sous le format « Nom, Nom (Base + suffixe)» (ex. couture, couturier). Les fausses paires sont construites sur le même schème (ex. saler, salade; colle, collier). Les paires de mots n'impliquent ni changement phonologique ni changement orthographique à l'ajout du suffixe. L'alpha de Cronbach pour cette épreuve est de 0,75 .

\subsubsection{Extraction de la base}


Cette tâche figurait initialement dans Rubin (1988) puis dans Colé et al. (2004) (mots et mots pseudo-affixés, comme fillette et alouette) et dans Reder, Marec-Breton, Gombert et Demont (2013), qui y ont ajouté des pseudo-mots affixés, comme tassette et dégarer. Elle est constituée de 24 items (Rubin, 1988; Colé et al., 2004; Reder, Marec-Breton, Gombert \& Demont, 2013), créés à partir de trois schèmes : d'abord, le schème base + affixe, afin de créer huit pseudo-mots, comme tassette ou bruitet, ensuite le schème base + affixe pour former huit mots existant bel et bien, comme fillette ou garçonnet, et enfin, le schème de mots pseudo-affixés, sur la base de alouette ou chalet. L'alpha de Cronbach pour cette épreuve est de 0,92 .

\subsubsection{Dérivation}

Dans le cadre de cette épreuve, qui n'est pas sans rappeler le fameux wug-test (Berko, 1958), l'élève doit mot compléter la phrase en ajoutant un suffixe au mot fourni (Carlisle, 1995). Le matériel pour la partie dérivationnelle comprend 16 phrases à trous, avec un mot à dériver pour chacune, comme dans l'exemple suivant : garage. Gaston est un [garagiste]. En tout, huit suffixes dérivationnels sont élicités (deux fois pour chacun d'eux). Les items se déclinent en quatre formats Base + Suffixe : ADJ $>N, N>N, N<$ ADJ et $\mathrm{V}>\mathrm{ADJ}$ (quatre items pour chaque format). Le matériel pour la partie flexionnelle comprend 8 phrases à trous, avec un mot à décomposer pour chacune. Deux types de flexions sont envisagées : de genre et de personne. Pour des questions de différenciation phonologique, nous n'avons gardé que des verbes conjugués à la première personne du pluriel, comme en témoigne cet exemple : écouter. Nous [écoutons]. Pour ce qui est des flexions en genre à décomposer, il s'agit de conjuguer au masculin ce qui était au féminin (ex : chanteuse-chanteur). Quatre types de suffixes sont présentés dans quatre mots, soit 2 adjectifs et deux noms : -esse $(\mathrm{N})$, ère $(\mathrm{N})$, -euse (Adj) et-ive (Adj). L'alpha de Cronbach pour cette épreuve est de 0,90 .

\subsubsection{Décomposition}

L'épreuve de décomposition comporte les mêmes consignes que l'épreuve de dérivation (Tighe et Binder, 2015, Carlisle, 2000, Kieffer, et al., 2016). Le matériel pour les items de morphologie dérivationnelle comprend 16 phrases à trous, avec un mot à décomposer pour chacune comme dans l'exemple qui suit: courageux. Mario a beaucoup de [courage]. En tout, 8 suffixes dérivationnels doivent être supprimés afin de retrouver la base (deux fois pour chacun d'eux) : -eur $(A D J>N)$, -ise $(A D J>N)$, -age $(N>N)$, -aire $(\mathrm{N}>\mathrm{N})$, -eux $(\mathrm{N}>\mathrm{ADJ})$, -ique $(\mathrm{N}>\mathrm{ADJ})$, - $(\mathrm{V}>\mathrm{ADJ})$, -atif $(\mathrm{V}>\mathrm{ADJ})$. Les items se déclinent en quatre formats Base + Suffixe : $\mathrm{ADJ}>\mathrm{N}, \mathrm{N}>\mathrm{N}, \mathrm{N}>\mathrm{ADJ}$ et $\mathrm{V}>\mathrm{ADJ}$. Il y a quatre items dans chaque format, pour un total de 16 items. Le matériel pour la partie flexionnelle comprend 8 phrases à trous, avec un mot à décomposer pour chacune. En concordance avec l'épreuve de dérivation, il s'agit d'accorder au masculin ce qui était au féminin (ex. fatigante. Mon chien est un peu épreuve de décomposition est de $0, \overline{75}$. [fatigant]). L'alpha de Cronbach pour notre

\subsubsection{Choix de pseudo-mots suffixés (morphèmes dérivationnels)}

Dans cette tâche, les participants doivent compléter une phrase en sélectionnant le pseudomot qui possède le suffixe dérivationnel approprié (Kieffer, et al., 2016; Nagy et al., 2006). On demande donc à l'élève de sélectionner la meilleure réponse parmi les choix offerts : ex. Hubert et son papa vont à la [ [louviterie ; louvitir ; louvitable]. Les suffixes qui ont été choisis pour construire la tâche sont tous exclusivement d'une catégorie grammaticale, c'est-à-dire qu'ils ne peuvent être utilisés que pour former des mots d'une 
seule catégorie grammaticale - adjectivale ou nominale, dans ce cas-ci (selon le Dictionnaire des suffixes du français le Robert). L'alpha de Cronbach pour cette épreuve est de 0,79 .

\subsubsection{Choix de pseudo-mots suffixés (morphèmes flexionnels)}

Les consignes de tâche de choix de pseudo-mots suffixés avec morphèmes sont les même que pour la tâche précédente, à la différence que quatre choix sont offerts, plutôt que trois. Dans le but de contrôler la complexité syntaxique de chacune des phrases, nous avons systématiquement construit des items où le verbe manquant est accompagné d'un autre verbe dans la phrase, comme on peut le constater dans l'exemple suivant : Lucie m'a dit de la porte. glodons ; gloder; glodant; glode. Chaque phrase comporte donc deux propositions (au sens de Riegel, 2004). L'alpha de Cronbach pour cette épreuve à choix de réponses est de 0,77 .

\subsubsection{Portrait global des épreuves et dimensions impliquées dans leur traitement}

Après avoir contrôlé certains facteurs pouvant influencer les résultats (notamment la fréquence, la transparence et les modalités de passation), il reste que les épreuves varient sur le plan des unités qui sont mises en jeu et sur les types de manipulations mobilisées. Le tableau 1 donne un aperçu des tâches et de leur contre-balancement sur le plan des trois différentes dimensions dont nous allons évaluer le pouvoir descriptif - l'unité, le type de contrôle et l'opération.

Tableau 1. Aperçu des tâches utilisées et de leur classement en fonction de trois dimensions

\begin{tabular}{|c|c|c|c|}
\hline Épreuves & Unité & $\begin{array}{l}\text { Type de } \\
\text { contrôle }\end{array}$ & Opération \\
\hline 1. Paires de mots & $\begin{array}{l}\text { Mots dérivés et pseudo-dérivés } \\
\text { avec suffixes dérivationnels }\end{array}$ & Implicite & Suppression \\
\hline 2. Extraction de la base & Suffixes dérivationnels & Explicite & Suppression \\
\hline 3. Dérivation & $\begin{array}{l}\text { Morphèmes dérivationnels et } \\
\text { flexionnels }\end{array}$ & Explicite & Ajout \\
\hline 4. Décomposition & $\begin{array}{l}\text { Morphèmes dérivationnels et } \\
\text { flexionnels }\end{array}$ & Explicite & Suppression \\
\hline $\begin{array}{l}\text { 5. Choix de pseudo- } \\
\text { mots suffixés D }\end{array}$ & Morphèmes dérivationnels & Implicite & Ajout \\
\hline $\begin{array}{l}\text { 6. Choix de pseudo- } \\
\text { mots suffixés F }\end{array}$ & Morphèmes flexionnels & Implicite & Ajout \\
\hline
\end{tabular}

\section{Présentation des résultats}

Nous avons d'abord analysé la distribution de nos variables, puis identifié les données aberrantes. Trois élèves dont les scores dans plus de trois épreuves étaient à trois mesures d'écart-type inférieures à la moyenne ont été repérés, puis nous les avons exclus de l'analyse (ce qui correspond à 3\% de perte dans l'échantillon). En suivant la même règle, 
deux données ont été supprimées puis remplacées par la valeur moyenne, afin d'obtenir des coefficients d'aplatissement et d'asymétrie acceptables (-1 à +1) (Howell, 2008).

\subsection{Statistiques descriptives}

Les résultats pour l'ensemble des groupes d'élèves figurent dans le tableau 2. Ceux-ci sont exprimés en pourcentages.

Tableau 2. Statistiques descriptives avec les pourcentages de réussite aux différentes épreuves

\begin{tabular}{llllll}
\hline Épreuves & N & Min & Max & Moyenne & Écart-type \\
\hline Paires de mots - D & 83 & 58.30 & 100.00 & 86.99 & 9.34 \\
Extraction de la base - D & 84 & 50.00 & 100.00 & 79.30 & 13.22 \\
Dérivation - D & 84 & 18.75 & 100.00 & 78.60 & 16.21 \\
Dérivation - F & 84 & 50.00 & 100.00 & 86.90 & 12.10 \\
Décomposition - D & 86 & 43.75 & 100.00 & 88.08 & 10.96 \\
Décomposition - F & 86 & 37.50 & 100.00 & 83.58 & 14.64 \\
Choix de pseudo-mots - D & 86 & 20.00 & 100.00 & 54.65 & 18.32 \\
Choix de pseudo-mots - F & 86 & 6.25 & 100.00 & 55.89 & 23.59 \\
\hline
\end{tabular}

On remarque que pour chaque épreuve, le maximum obtenu correspond à une note parfaite de $100 \%$. La variabilité dans les résultats est toutefois évidente (cf. moyennes et écarts types) : il n'y a pas d'effet plafond.

Tableau 3. Corrélations entre les différentes tâches

$\begin{array}{llllllll}\text { A } & \text { B } & \text { C } & \text { D } & \text { E } & \text { F } & \text { G } & \text { H }\end{array}$
A. Paires mots - D 1
B. Extract. base - D $\quad .069 \quad 1$
C. Dérivation - D $\quad .364 * * \quad .006 \quad 1$
D. Dérivation - F $\quad .326 * * \quad .023 \quad .501 * * \quad 1$
E. Décomp. - D $\quad .235^{*} \quad .020 \quad .384 * * \quad .318^{* *} \quad 1$
F. Décomp. - F $\quad .321 * * \quad .110 \quad .399 * * \quad .279 * \quad .467 * * \quad 1$
$\begin{array}{llllllll}\text { G. Choix PM - D } & .287 * * & -.008 & .416^{* *} & .373^{* *} & .372 * * & .221 * & 1\end{array}$

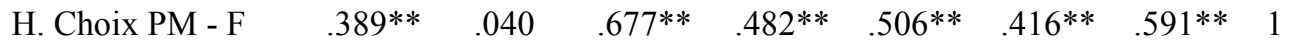

Des analyses de corrélation de Pearson ont été effectuées, afin de voir si les épreuves, qui $a$ priori mesurent le même construit théorique, étaient fortement corrélées. Comme les analyses figurant dans le tableau 3 le montrent, les corrélations sont moyennes et même 
plutôt faibles en général, ce qui suggère que les épreuves ne sont pas équivalentes : dans la majorité des cas, le coefficient de corrélation $r$ est sous 0,4 . Il s'agit d'un résultat somme toute étonnant, dans la mesure où ces tâches sont régulièrement utilisées de façon interchangeable dans la littérature pour mesurer le même construit (Apel, 2014).

Le tableau 3 montre que l'épreuve d'extraction de la base (voir colonne B dans le tableau 3) n'est significativement corrélée avec aucune autre. Cela peut être causé par une mauvaise compréhension des tâches par les enfants, et/ou des instructions imprécises malgré l'utilisation d'un même protocole de passation. L'épreuve d'extraction de la base a tout de même été maintenue dans les analyses subséquentes, puisque les analyses factorielles confirmatoires, qui permettent d'extraire la variance commune dans ces variables mesurées à validité plus faible, sont robustes face à ce type de problème (Spencer et al., 2015).

\subsection{Analyses factorielles confirmatoires}

Pour répondre à notre question de recherche concernant la dimensionnalité de la conscience morphologique, une série de quatre analyses factorielles confirmatoires ont été effectuées à l'aide du logiciel Amos® (Arbuckle, 2014). L'analyse factorielle confirmatoire, qui fait partie de la famille des modèles à équations structurelles (Structural Equation Modeling), est utilisée pour identifier des variables latentes à partir de certaines variables mesurées, tout en permettant de spécifier des corrélations entre les variables latentes et d'indiquer des erreurs résiduelles. Les variables latentes dans les modèles spécifiés dans le cadre des analyses factorielles confirmatoires correspondent aux dimensions sous-jacentes aux épreuves de conscience morphologique que nous avons délimitées - unité, type de contrôle et opération.

Le bon ajustement d'un modèle aux données d'analyse factorielle confirmatoire indique que l'on a réussi à identifier un modèle théorique qui circonscrit bien le phénomène à l'étude. Une analyse des mêmes données avec un autre modèle peut donner de meilleurs résultats. Aussi faut-il tester plusieurs modèles et les comparer pour identifier lequel représente le mieux les données en question. Il est donc légitime, lorsqu'on utilise ce type d'analyse, de confronter plusieurs modèles, pour trouver celui qui s'ajuste le mieux aux données recueillies. 


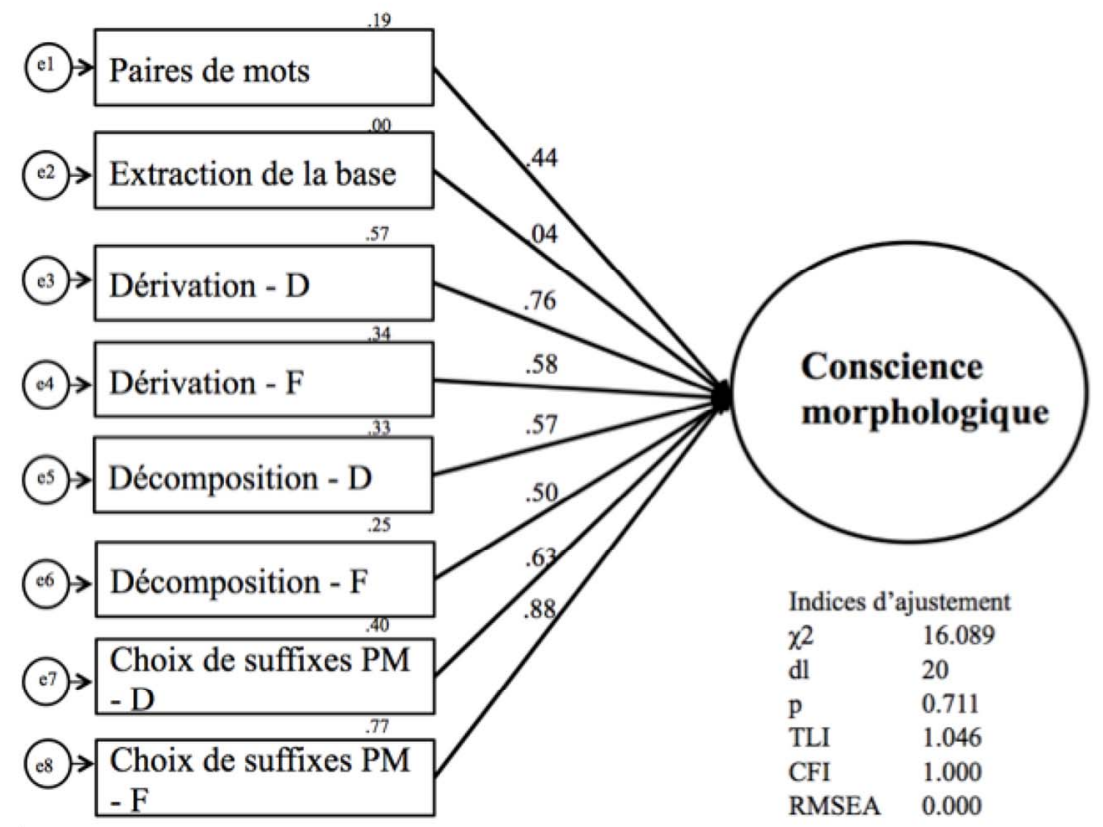

Fig. 1. Modèle unidimensionnel de la conscience morphologique (modèle A).

Le premier modèle spécifié (modèle A) propose de penser la conscience morphologique comme facteur à une dimension. Les trois modèles testés subséquemment au modèle unidimensionnel sont bidimensionnels : l'un suggère que les épreuves construisent deux types d'unités (morphèmes flexionnels ou dérivationnels) (modèle B), l'autre, deux types de traitement (implicite ou explicite) (modèle C) et le dernier, repris dans la figure 2, deux types d'opérations cognitives (ajout ou suppression) (modèle D).

Nous avons donc utilisé un modèle unidimensionnel de conscience morphologique, où toutes les tâches font appel à une seule dimension. Le modèle A fournit un bon ajustement aux données, comme le suggèrent les indices d'ajustement du modèle, fournis dans la figure 1 , ce qui indique que le modèle spécifié s'applique au phénomène; il le circonscrit bien (Harrington, 2009).

Nous avons réalisé un deuxième modèle (modèle $\mathrm{B}$ ), dans lequel il est proposé que le type d'unité impliquée dans les tâches, soit les morphèmes dérivationnels ou flexionnels, représente les différences individuelles dans la qualité des réponses. Puisque le modèle unidimensionnel est imbriqué (nested) dans les modèles bidimensionnels, il est possible d'évaluer la significativité de leur différence avec un test de chi-deux. Le modèle fournit un bon ajustement aux données, mais il n'est pas significativement meilleur que l'unidimensionnel [différence de chi-deux : 3,721(1), $\mathrm{p}=0,053$ ].

Un modèle bidimensionnel de conscience morphologique (modèle C), distinguant les tâches implicites des tâches explicites a ensuite été testé et s'ajustait également bien aux données sans supplanter le modèle unidimensionnel sur le plan explicatif [différence de chi-deux : $0,815(1), \mathrm{p}=0,367]$. 


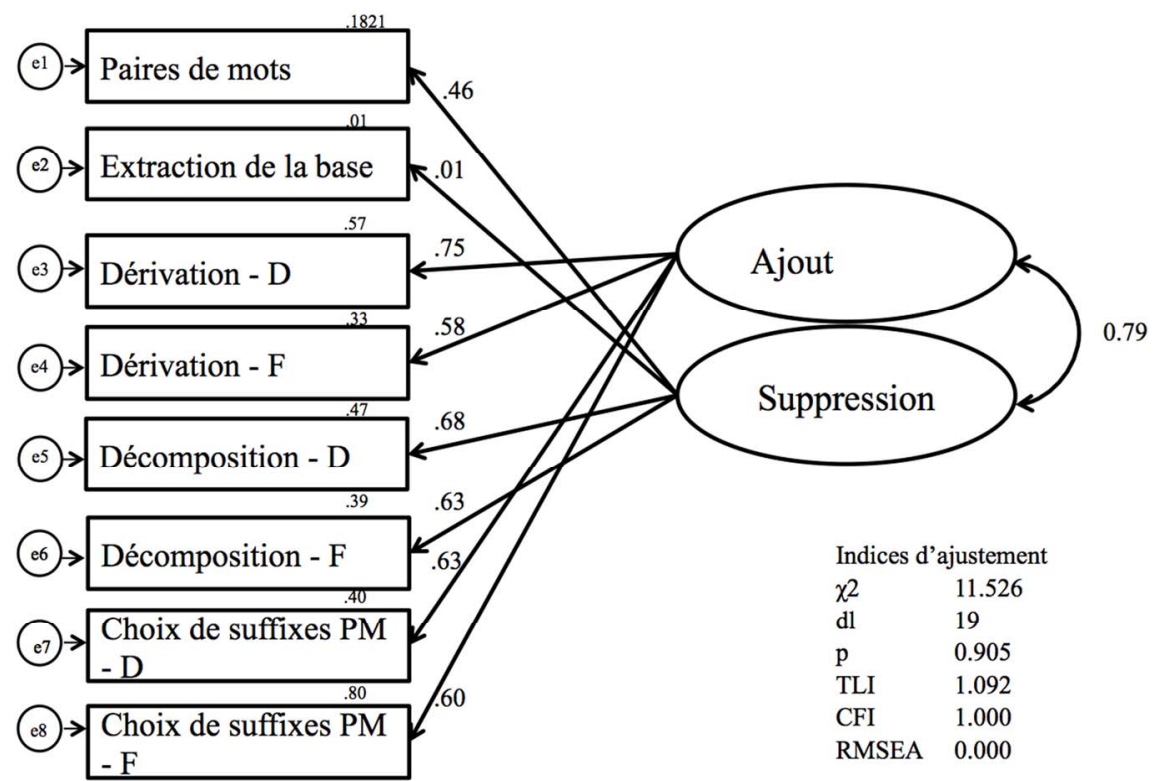

Fig. 2. Modèle bidimensionnel (modèle D)

Les résultats de l'analyse factorielle confirmatoire fournis par le modèle séparant les épreuves en deux types d'opérations correspondent mieux aux données que le modèle $\mathrm{D}$ unidimensionnel [différence de chi-deux : 4,494(1), $\mathrm{p}=0,03$ ]. Ce modèle semble donc être le meilleur, parmi les modèles testés, pour représenter nos données.

\section{Discussion}

Les résultats des analyses factorielles confirmatoires montrent la manière dont les différentes tâches se regroupent et permettent de brosser un tableau plus clair des capacités mobilisées lors des tâches traditionnellement utilisées pour mesurer la conscience morphologique. Nous avons d'abord proposé un modèle unidimensionnel, dans lequel était posée l'hypothèse de la construction d'une seule habileté, la conscience morphologique, par toutes les épreuves, puis trois modèles bidimensionnels. Les analyses nous permettent de conclure que les tâches réputées mesurer la conscience morphologique circonscrivent bel et bien ce construit théorique, malgré leurs différences sur le plan de l'unité traitée et du type de traitement.

Il appert que la conscience morphologique est pourtant significativement mieux représentée par un modèle à deux facteurs impliquant deux opérations cognitives : l'addition et la suppression. En effet, c'est ce modèle bidimensionnel de conscience morphologique, avec les tâches délimitées en fonction des opérations qu'elles impliquent, qui s'ajuste le mieux aux données et permet mieux de représenter les différences individuelles. Les résultats ont des implications pratiques pour les chercheurs évaluant la conscience morphologique, car ils indiquent que différentes épreuves de conscience morphologique exploitent des dimensions différentes du même construit (Apel et al., 2013; Apel, 2014; Tighe et Schatschneider, 2015).

Notre objectif de recherche était de faire ressortir les dimensions sous-jacentes des tâches utilisées traditionnellement pour évaluer la conscience morphologique. Bien que plusieurs études concluent à l'unidimensionnalité de la conscience morphologique, même en 
mesurant neuf épreuves (Muse, 2005) ou en proposant des modèles alternatifs (Spencer et al. 2015), notre recherche apporte sa pierre à l'édifice d'une conception multidimensionnelle de la conscience morphologique. Tibi et Kirby (2017) proposent que le construit peut être envisagé en fonction de deux sous-composantes, les modalités orale et écrite, et les résultats de Tighe et Schatschneider (2015) indiquent, eux, que la multidimensionnalité de la conscience morphologique peut être représentée par la nature de l'unité mise en jeu, soit les vrais et les pseudo mots. Nous avons, pour notre part, rajouté le type d'opérations mises en jeu dans les épreuves de conscience morphologique. Nos résultats montrent clairement que la prise en compte de cet aspect amène un gain significatif dans l'explication de la variabilité dans les résultats. A notre connaissance, les opérations, telles que définies dans cet article, n'avaient pas été prises en compte en ellesmêmes dans les travaux précédents sous l'angle de la validité théorique (construct validity), bien que la conscience morphologique soit définie comme la manipulation consciente d'unités morphologiques. Or il nous semble pourtant que cette dimension, qui prend en compte le type d'opérations, est constitutive du construit théorique tel que défini dans la littérature sur la conscience morphologique (Carlisle, 1995).

\section{Conclusion}

Les analyses effectuées dans le cadre de cette recherche permettent de tirer quelques réponses sur la dimensionnalité de la conscience morphologique. Tel que prévu, les tâches utilisées mobilisent des habiletés extrêmement variées : la conscience morphologique peut être comprise comme un construit multidimensionnel dans lequel les opérations d'ajout et de suppression jouent un rôle prépondérant. Les tâches de conscience morphologique reposent sur différentes dimensions sous-jacentes qui permettent leur réussite, c'est-à-dire que la mobilisation de différentes capacités opérationnelles permet de répondre différemment aux épreuves de conscience morphologique. Cette constatation est fondamentale. Il faut se demander comment cet état de choses peut avoir une résonance dans d'autres habiletés linguistiques. Dans la mesure où ces opérations peuvent certainement être impliquées dans certaines autres tâches métalinguistiques, il s'avère indispensable d'évaluer la part que représentent les opérations cognitives et la part que représente l'unité linguistique manipulée dans la réussite à ces différentes épreuves.

La sophistication des analyses statistiques utilisées et les résultats dont elles rendent compte ne doivent pas occulter l'importance du travail qu'a représenté la confection du matériel expérimental et la systématicité des contre-balancements dans la construction des items, travail grâce auquel plusieurs variables confondantes ont été contrôlées (notamment la fréquence lexicale, la modalité de passation (input/output), la catégorie grammaticale des items et la transparence). C'est cette phase indispensable qui nous permet d'en arriver avec conviction à cet état des lieux.

Plusieurs limites doivent être mentionnées en regard de la présente étude, afin d'être incluses dans les démarches expérimentales de futures recherches. Il y a lieu de se demander d'abord jusqu'à quel point la qualité de la réponse, notée ici et dans plusieurs études de façon binaires, est réellement représentative des habiletés sur le plan de la conscience morphologique. Il conviendrait d'examiner les temps de réponse, et sans doute de les considérer comme une mesure plus fiable de la conscience morphologique. En effet, puisque les temps de réponse sont un indicateur de l'automaticité des processus, ceux-ci pourraient indiquer plus précisément ce que c'est que d'avoir une bonne conscience morphologique, et mieux représenter la variation individuelle. De plus, notre analyse aurait gagné à être étendue à d'autres dimensions constitutives des épreuves (notamment les suivantes : mots vs pseudo-mots et indices contextuels $v s$ aucun indice contextuel, cf. Tighe 
et Schatschneider, 2015). Pour finir, à l'instar d'autres études s'intéressant à la spécificité de la conscience morphologique, il serait important à l'avenir de mesurer et de contrôler l'impact du vocabulaire sur les données dans le plan expérimental (Spencer et al. 2015 ; Tibi et Kirby, 2017). La conscience morphologique doit être distinguée de la connaissance $\mathrm{du}$ vocabulaire, puisque ces deux habiletés ne sont pas mutuellement exclusives. Cependant, nous avons systématiquement contrôlé cet aspect dans la constitution de notre matériel expérimental.

Les orientations futures découlant de cette recherche doivent au moins en partie porter sur une réflexion quant au pouvoir prédictif différencié des épreuves de conscience morphologique vis-à-vis des habiletés complexes que sont par exemple la lecture et l'écriture. En effet, ces divergences pourraient expliquer les résultats distincts que rapportent les études (Spencer et al., 2015). Dans le but d'examiner la contribution de différentes dimensions, ou facteurs latents, relatives à la conscience morphologique à la compréhension en lecture, il y aurait lieu de réaliser des analyses avec des modèles d'équations structurelles.

Sachant que beaucoup de tâches de conscience métalinguistique impliquent des opérations comme celles que nous avons pu observer dans le cadre de notre recherche (Gombert, 1990; Gombert, Gaux et Demont, 1994; Casalis et al., 2003; Desrochers, Kirby, Thomson et Fréchette, 2009; Berthiaume, Besse et Daigle, 2010), nous pourrions penser que la compréhension en lecture implique de telles habiletés cognitives. Cette réflexion suggère qu'un construit aussi complexe que la compréhension en lecture pourrait être en partie expliqué par la variation sur le plan de l'automatisation d'habiletés cognitives permettant de supprimer et d'ajouter des éléments linguistiques. Ainsi, en délimitant de façon systématique les opérations cognitives impliquées dans les épreuves, il devient possible de mieux comprendre quelles dimensions linguistiques et cognitives sont plus importantes pour réussir dans certaines compétences essentielles au succès scolaire.

Les auteur-e-s tiennent à remercier les évaluateurs et évaluatrices pour leurs commentaires et apports constructifs sur une version antérieure de cet article.

\section{Références}

Allard, D. (2008). Connaissances lexicales et en morphologie dérivationnelle chez des apprenants adultes avancés en français langue seconde. Université du Québec à Montréal, Montréal.

Antidote 9 [Logiciel]. (2017). Montréal, Druide informatique.

Apel, K. (2014). A Comprehensive Definition of Morphological Awareness. Topics in Language Disorders, 34(3), 197-209.

Apel, K., Diehm, E., \& Apel, L. (2013). Using Multiple Measures of Morphological Awareness to Assess its Relation to Reading. Topics in Language Disorders, 33(1), 42-56.

Arbuckle, J. L. (2017). Amos (Version 24.0) [Computer Program]. Chicago: IBM SPSS.

Berko, J. (1958). The child's learning of English morphology. Word, 14, 150-177. 
Berthiaume, R., Besse, A.-S., \& Daigle, D. (2010). L'évaluation de la conscience morphologique: proposition d'une typologie des tâches. Language Awareness, 19(3), 153-170.

Bloomfield, Leonard. (1933). Language. New York: Henry Holt.

Carlisle, J. F. (1995). Morphological awareness and early reading achievement. Hillsdale: NJ: Erlbaum.

Carlisle, J. F. (2000). Awareness of the structure and meaning of morphologically complex words: Impact on reading. Reading and Writing: An Interdisciplinary Journal, 12, 169-190.

Carlisle, J. F. (2003). Morphology matters in learning to read: A commentary. Reading Psychology, 24, 291-332.

Carlisle, J.F. et Nomanbhoy, D.M. (1993). Phonological and morphological awareness in first graders. Applied Psycholinguistics, 14, 177-195.

Carlisle, J. F., \& Stone, C. A. (2005). Exploring the role of morphemes in word reading. Reading Research Quarterly, 40(4), 428-449.

Casalis, S., \& Louis-Alexandre, M.-F. (2000). Morphological analysis, phonological analysis and learning to read French: a longitudinal study. Reading and Writing: An Interdisciplinary Journal, $12,303-335$.

Casalis, S., Mathiot, E., Bécavin, A-S., et Colé, P. (2003). Conscience morphologique chez des apprentis lecteurs tout-venant et en difficultés. In : Fradin B. et al. (dir.), Silexicales $n^{\circ} 3$, « Les unités morphologiques », Actes du 3e Forum de morphologie de Villeneuve d'Ascq, 19-21 septembre 2002, 57-66.

Chomsky, N. \& Halle, M. (1968). The Sound Pattern of English. New York: Harper \& Row.

Colé, P., Royer, C., Leuwers, C., \& Casalis, S. (2004). Les connaissances morphologiques dérivationnelles et l'apprentissage de la lecture chez l'apprenti-lecteur français du CP au CE2. L'année psychologique, 104(4), 701-750.

Deacon, S. H., Parrila, R., \& Kirby, J. R. (2008). A review of the evidence on morphological processing in dyslexics and poor readers: A strength of weakness? Learning, Cognition and Dyslexia. 192-211.

Desrochers, A., Kirby, J. R., Thompson, G. L., \& Fréchette, S. (2009). Le rôle de la conscience phonologique dans l'apprentissage de la lecture. Revue du Nouvel-Ontario,(34), 59.

Duncan, L.G., Casalis, S. et Colé, P. (2009). Early metalinguistic awareness of derivational morphology: Observations from a comparison of English and French. Applied Psycholinguistics, 30(3), 405 .

Foucambert, D., \& Bourdages, R., (2017). Logix@ (version 1.0) [Logiciel informatique], Montréal.

Gombert, J. É. (1990). Le développement métalinguistique. Paris: Presses Universitaires de France.

Gombert, J. É., Gaux, C., \& Demont, E. (1994). Capacités métalinguistiques et lecture : quels liens ? Repères, 9, 61-73.

Green, L., D., McCutchen, C., Schwiebert, T., Quinlan, A., Eva-Wood, A., \& Juelis, J. (2003). Morphological development in children's writing. Journal of Educational Psychology, 95(4), $452-761$.

Harrington, D. (2009). Confirmatory factor analysis, Toronto : Oxford University Press. 
Howell, D. C. (2008). Méthodes statistiques en sciences humaines, 2e édition (D. Boeck Ed.).

Kieffer, M.J., Petscher, Y., Proctor, C.P. \& Silverman, R.D. (2016). Is the Whole Greater Than the Sum of Its Parts? Modeling the Contributions of Language Comprehension Skills to Reading Comprehension in the Upper Elementary Grades. Scientific Studies of Reading, 1-19.

Kuo, L.-J., \& Anderson, R. C. (2006). Morphological Awareness and Learning to Read: A CrossLanguage Perspective. Educational Psychologist, 41(3), 161-180.

Le Petit Robert. Dictionnaire alphabétique et analogique de la langue française, nouvelle édition millésime 2013, Paris, Dictionnaires Le Robert, "Dictionnaire des suffixes du français le Robert », accessible au http://robert-illustre.lerobert.com/pdf/dictionnaire-des-suffixes.pdf.

Manolitsis, G., Grigorakis, I., \& Georgiou, G. K. (2017). The Longitudinal Contribution of Early Morphological Awareness Skills to Reading Fluency and Comprehension in Greek. Frontiers in Psychology, 1-32.

Muse, A. E. (2005). The nature of morphological knowledge. Florida State University, Tallahassee.

Nagy, W., Berninger, V. W., \& Abbott, R. D. (2006). Contributions of Morphology Beyond Phonology to Literacy Outcomes of Upper Elementary and Middle-School Students. Journal of Educational Psychology, 98(1), 134-147.

Reder, F., Marec-Breton, N., Gombert, J. E., \& Demont, E. (2013). Second-language learners' advantage in metalinguistic awareness: a question of languages' characteristics. British Journal of Educational Psychology, 83(4), 686-702.

Riegel, R. Pellat, J-C., Rioul, R. (2004). Grammaire méthodique du français. Payot.

Roy, C. et Labelle, M. (2007). Connaissance de la morphologie dérivationnelle chez les francophones et non-francophones de 6 à 8 ans. Revue canadienne de linguistique appliquée, 263-291.

Rubin, H. (1988). Morphological Knowledge and Early Writing Ability. Language and Speech, 31(4), $337-355$.

Spencer, M., Muse, A., Wagner, R. K., Foorman, B., Petscher, Y., Schatschneider, C., Tighe, Elizabeth L. \& Bishop, M. D. (2015). Examining the underlying dimensions of morphological awareness and vocabulary knowledge. Reading and Writing, 28(7), 959-988.

Tibi, S., \& Kirby, J. R. (2017). Morphological awareness: Construct and predictive validity in Arabic. Applied Psycholinguistics, 38(5), 1019-1043.

Tighe, E. L., \& Binder, K. S. (2015). An investigation of morphological awareness and processing in adults with low literacy. Applied Psycholinguistics, 36(2), 245-273.

Tighe, E. L., \& Schatschneider, C. (2015). Exploring the Dimensionality of Morphological Awareness and Its Relations to Vocabulary Knowledge in Adult Basic Education Students. Reading Research Quarterly, 50(3), 293-311.

Tighe, E. L., \& Schatschneider, C. (2016). Modeling the Relations Among Morphological Awareness Dimensions, Vocabulary Knowledge, and Reading Comprehension in Adult Basic Education Students. Frontiers in Psychology, 7, 86.

Tyler, A., \& Nagy, W. (1989). The acquisition of English derivational morphology. Journal of Memory and Language, 28(6), 649-667. 